\title{
WIDE SUBCATEGORIES AND LATTICES OF TORSION CLASSES
}

\author{
SOTA ASAI AND CALVIN PFEIFER
}

\begin{abstract}
In this paper, we study the relationship between wide subcategories and torsion classes of an abelian length category $\mathcal{A}$ from the point of view of lattice theory. Motivated by $\tau$-tilting reduction of Jasso, we mainly focus on intervals $[\mathcal{U}, \mathcal{T}]$ in the lattice tors $\mathcal{A}$ of torsion classes in $\mathcal{A}$ such that $\mathcal{W}:=\mathcal{U}^{\perp} \cap \mathcal{T}$ is a wide subcategory of $\mathcal{A}$; we call these intervals wide intervals. We prove that a wide interval $[\mathcal{U}, \mathcal{T}]$ is isomorphic to the lattice tors $\mathcal{W}$ of torsion classes in the abelian category $\mathcal{W}$. We also characterize wide intervals in two ways: First, in purely lattice theoretic terms based on the brick labeling established by Demonet-Iyama-Reading-Reiten-Thomas; and second, in terms of the Ingalls-Thomas correspondences between torsion classes and wide subcategories, which were further developed by Marks-Šťovíček.
\end{abstract}

\section{Contents}

1. Introduction

2. Setting and notation

2.1. Partially ordered sets

2.2. Abelian length categories

3. Brick labeling

4. Wide intervals and reduction of torsion classes

5. Classification of wide intervals in terms of join and meet intervals

6. Classification of wide intervals in terms of Ingalls-Thomas correspondences

7. Widely generated torsion classes

Funding

Acknowledgement

References

\section{INTRODUCTION}

In the mid-20th century Dickson Dic vastly generalized the torsion theory of abelian groups to abelian categories $\mathcal{A}$. He defined a torsion pair $(\mathcal{T}, \mathcal{F})$ in $\mathcal{A}$ to be a pair of full subcategories $\mathcal{T}, \mathcal{F} \subseteq \mathcal{A}$ satisfying the Hom-orthogonality conditions

$$
\mathcal{F}=\mathcal{T}^{\perp} \text { and } \mathcal{T}={ }^{\perp} \mathcal{F} \text {. }
$$

Here and throughout, we indicate the right Hom-perpendicular category of a full subcategory $\mathcal{X} \subseteq \mathcal{A}$ by

$$
\mathcal{X}^{\perp}:=\left\{Y \in \mathcal{A} \mid \text { for all } X \in \mathcal{X}, \operatorname{Hom}_{\mathcal{A}}(X, Y)=0\right\},
$$

and define the left Hom-perpendicular category ${ }^{\perp} \mathcal{X}$ dually. Since then many authors investigated torsion pairs from several points of view, including their classification

Date: February 25, 2020. 
Brü, Hov, derived equivalences [BB, Hap, HRS, Ric, cluster theory [IT], $\tau$-tilting theory AIR, KY, MS and stability conditions Bri1.

From now on we assume that $\mathcal{A}$ is an essentially small abelian length category. For a full subcategory $\mathcal{T} \subseteq \mathcal{A}$ there is a torsion pair $(\mathcal{T}, \mathcal{F})$ in $\mathcal{A}$ if and only if $\mathcal{T}$ is closed under extensions and factor objects; such subcategories $\mathcal{T}$ are called torsion classes. In our setting the torsion classes in $\mathcal{A}$ form a set tors $\mathcal{A}$, partially ordered by inclusion. Note that tors $\mathcal{A}$ is stable under arbitrary intersections. In particular, for every full subcategory $\mathcal{X} \subseteq \mathcal{A}$, there is a smallest torsion class $\mathrm{T}(\mathcal{X})$ containing $\mathcal{X}$, namely

$$
\mathrm{T}(\mathcal{X}):=\bigcap_{\mathcal{T} \in \text { tors } \mathcal{A} ; \mathcal{X} \subseteq \mathcal{T}} \mathcal{T}
$$

It is not hard to see that the partially ordered set tors $\mathcal{A}$ is in fact a complete lattice, that is, joins and meets of arbitrary subsets of tors $\mathcal{A}$ exist. More generally, every interval

$$
[\mathcal{U}, \mathcal{T}]:=\{\mathcal{V} \in \text { tors } \mathcal{A} \mid \mathcal{U} \subseteq \mathcal{V} \subseteq \mathcal{T}\}
$$

in tors $\mathcal{A}$ is a complete lattice. To an interval $[\mathcal{U}, \mathcal{T}]$ in tors $\mathcal{A}$ we associate a full subcategory $\mathcal{W}:=\mathcal{U}^{\perp} \cap \mathcal{T}$ as proposed in DIRRT. This subcategory "measures the difference" between $\mathcal{U}$ and $\mathcal{T}$ in a precise sense (see Lemma 3.1). We investigate those intervals $[\mathcal{U}, \mathcal{T}]$ in tors $\mathcal{A}$ such that $\mathcal{W}:=\mathcal{U}^{\perp} \cap \mathcal{T}$ is an abelian subcategory of $\mathcal{A}$; that is, $\mathcal{W}$ is closed under kernels, images and cokernels. Then we can define the complete lattice tors $\mathcal{W}$ as before and compare it with the interval $[\mathcal{U}, \mathcal{T}]$ (see Theorem 1.4). Note that such subcategories $\mathcal{W}$ are always closed under extensions, as $\mathcal{U}^{\perp}$ and $\mathcal{T}$ are. Thus, if we assume that $\mathcal{W}$ is abelian, it will be automatically a wide subcategory of $\mathcal{A}$, that is, an abelian subcategory closed under extensions. This motivates the following definition.

Definition 1.1 (Definition 4.1). We call an interval $[\mathcal{U}, \mathcal{T}]$ in tors $\mathcal{A}$ a wide interval if $\mathcal{U}^{\perp} \cap \mathcal{T}$ is a wide subcategory of $\mathcal{A}$.

An important class of examples of wide intervals is given by numerical torsion classes in the case $\mathcal{A}=\bmod A$, where $A$ is a finite-dimensional algebra over a field $K$. Let us write $\operatorname{proj} A$ for the category of finitely generated projective $A$-modules.

Proposition 1.2. BKT, Bri2 For each element $\theta \in K_{0}(\operatorname{proj} A) \otimes_{\mathbb{Z}} \mathbb{R}$, define two numerical torsion classes $\mathcal{T}_{\theta} \subseteq \overline{\mathcal{T}}_{\theta}$ in $\bmod A$ by

$$
\begin{aligned}
\mathcal{T}_{\theta} & :=\{M \in \bmod A \mid \text { for any nonzero quotient } N, \theta(N)>0\}, \\
\overline{\mathcal{T}}_{\theta} & :=\{M \in \bmod A \mid \text { for any quotient } N, \theta(N) \geq 0\} .
\end{aligned}
$$

Then $\left[\mathcal{T}_{\theta}, \overline{\mathcal{T}}_{\theta}\right]$ is a wide interval with the associated wide subcategory $\mathcal{W}_{\theta}:=\left(\mathcal{T}_{\theta}\right)^{\perp} \cap$ $\overline{\mathcal{T}}_{\theta}$ is the $\theta$-semistable subcategory defined by King [Kin.

See also BST, Yur, Asa2 for more information on numerical torsion classes.

Another important example appears in $\tau$-tilting reduction as developed by Jasso Jas].

Proposition 1.3. Jas, Theorem 3.12] For a $\tau$-rigid module $U \in \bmod A$, the interval $\left[\mathrm{Fac} U,^{\perp}(\tau U)\right]$ is a wide interval. This interval is isomorphic to the lattice tors $\mathcal{W}_{U}$ of torsion classes in $\mathcal{W}_{U}:=U^{\perp} \cap^{\perp}(\tau U)$.

We aim to extend this result in this paper.

In general, we call two torsion classes $\mathcal{U} \subsetneq \mathcal{T}$ in $\mathcal{A}$ adjacent if there exists no torsion class $\mathcal{V}$ in $\mathcal{A}$ satisfying $\mathcal{U} \subsetneq \mathcal{V} \subsetneq \mathcal{T}$. For example, if $[\mathcal{U}, \mathcal{T}]=\left[\operatorname{Fac} U,{ }^{\perp}(\tau U)\right]$ and $U$ is an almost support $\tau$-tilting module, then $\mathcal{U}$ and $\mathcal{T}$ are adjacent DIJ, Example 3.5]. For two adjacent torsion classes $\mathcal{U} \subsetneq \mathcal{T}$, the subcategory $\mathcal{W}=\mathcal{U}^{\perp} \cap \mathcal{T}$ 
is wide and contains a unique brick $S$ [BCZ, DIRRT. In particular, we can label the arrow $\mathcal{T} \rightarrow \mathcal{U}$ in the Hasse quiver Hasse(tors $\mathcal{A}$ ) of the partially ordered set tors $\mathcal{A}$ by the brick $S$. This is called brick labeling of Hasse(tors $\mathcal{A}$ ) introduced in Asa1 for Hasse arrows between functorially finite torsion classes and in general in DIRRT.

Our first result shows that the wide interval $[\mathcal{U}, \mathcal{T}]$ and tors $\mathcal{W}$ are isomorphic complete lattices and that this isomorphism is compatible with their brick labelings.

Theorem 1.4 (Theorem 4.2). Let $[\mathcal{U}, \mathcal{T}]$ be a wide interval in tors $\mathcal{A}$ and set $\mathcal{W}:=$ $\mathcal{U}^{\perp} \cap \mathcal{T}$.

(1) There are mutually inverse isomorphisms of complete lattices

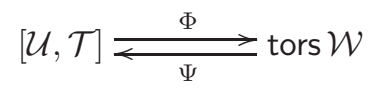

given by $\Phi(\mathcal{V}):=\mathcal{U}^{\perp} \cap \mathcal{V}$ and $\Psi(\mathcal{X}):=\mathrm{T}(\mathcal{U}, \mathcal{X})$ for $\mathcal{V} \in[\mathcal{U}, \mathcal{T}]$ and $\mathcal{X} \in$ tors $\mathcal{W}$ respectively. Moreover, $\Psi(\mathcal{X})=\mathcal{U} * \mathcal{X}$ holds for every $\mathcal{X} \in$ tors $\mathcal{W}$.

(2) The isomorphism $\Phi$ preserves the brick labeling: Namely, the brick label of $\mathcal{V}_{1} \rightarrow \mathcal{V}_{2}$ in $[\mathcal{U}, \mathcal{T}]$ is the same as the brick label of $\Phi\left(\mathcal{V}_{1}\right) \rightarrow \Phi\left(\mathcal{V}_{2}\right)$ in tors $\mathcal{W}$.

(3) The following sets coincide:

- The set $\operatorname{sim} \mathcal{W}$ of isomorphism classes of simple objects in $\mathcal{W}$.

- The set of labels of arrows in the Hasse quiver of $[\mathcal{U}, \mathcal{T}]$ starting at $\mathcal{T}$.

- The set of labels of arrows in the Hasse quiver of $[\mathcal{U}, \mathcal{T}]$ ending at $\mathcal{U}$.

This is a large extension of [Jas, Theorem 3.12] and [DIRRT, Theorem 4.12, Proposition 4.13, Theorem 4.16], which deal with functorially finite torsion classes in $\bmod A$.

Thus, it is natural to characterize wide intervals in terms of Hasse arrows in tors $\mathcal{A}$. For this purpose, we define

$$
\begin{aligned}
& {[\mathcal{U}, \mathcal{T}]^{+}:=\{\mathcal{T}\} \cup\{\mathcal{V} \in[\mathcal{U}, \mathcal{T}] \mid \text { there exists }(\mathcal{T} \rightarrow \mathcal{V}) \in \text { Hasse }(\text { tors } \mathcal{A})\}} \\
& {[\mathcal{U}, \mathcal{T}]^{-}:=\{\mathcal{U}\} \cup\{\mathcal{V} \in[\mathcal{U}, \mathcal{T}] \mid \text { there exists }(\mathcal{V} \rightarrow \mathcal{U}) \in \text { Hasse }(\text { tors } \mathcal{A})\}}
\end{aligned}
$$

and introduce the following two lattice theoretic notions for intervals in tors $\mathcal{A}$ :

Definition 1.5 (Definition $[5.1)$. Let $[\mathcal{U}, \mathcal{T}]$ be an interval in tors $\mathcal{A}$.

(1) We call $[\mathcal{U}, \mathcal{T}]$ a join interval if $\mathcal{T}=\bigvee[\mathcal{U}, \mathcal{T}]^{-}$.

(2) We call $[\mathcal{U}, \mathcal{T}]$ a meet interval if $\mathcal{U}=\bigwedge[\mathcal{U}, \mathcal{T}]^{+}$.

Our second main result characterizes wide intervals as precisely the join and the meet intervals.

Theorem 1.6 (Theorem 5.2). Let $[\mathcal{U}, \mathcal{T}]$ be an interval in tors $\mathcal{A}$. The following conditions are equivalent:

(a) The interval $[\mathcal{U}, \mathcal{T}]$ is a wide interval.

(b) The interval $[\mathcal{U}, \mathcal{T}]$ is a join interval.

(c) The interval $[\mathcal{U}, \mathcal{T}]$ is a meet interval.

Apart from wide intervals, there are other operations connecting torsion classes and wide subcategories. First, the operation $T$ given in (1.1) defines a map from the set wide $\mathcal{A}$ of wide subcategories in $\mathcal{A}$ to tors $\mathcal{A}$. On the other hand, IngallsThomas [T] and Marks-Štovíček MS introduced a map $\mathrm{W}_{\mathrm{L}}:$ tors $\mathcal{A} \rightarrow$ wide $\mathcal{A}$ (see (6.1) for the definition) such that the composite $\mathrm{W}_{\mathrm{L}} \circ \mathrm{T}$ is the identity. It is therefore important to understand the torsion classes in the image of the map $\mathrm{T}:$ wide $\mathcal{A} \rightarrow$ tors $\mathcal{A}$, which we call widely generated torsion classes. 
For this purpose, we will first prove that the wide subcategory $\mathrm{W}_{\mathrm{L}}(\mathcal{T})$ is related to wide intervals as follows. Below, Serre $\left(\mathrm{W}_{\mathrm{L}}(\mathcal{T})\right)$ denotes the set of Serre subcategories of $\mathrm{W}_{\mathrm{L}}(\mathcal{T})$.

Theorem 1.7 (Theorem 6.7). Let $\mathcal{T} \in$ tors $\mathcal{A}$. Taking labels gives a bijection

$\{$ Hasse arrows in tors $\mathcal{A}$ starting at $\mathcal{T}\} \rightarrow \operatorname{sim}\left(\mathrm{W}_{\mathrm{L}}(\mathcal{T})\right)$.

Moreover, the map $\mathcal{W} \mapsto \mathcal{T} \cap{ }^{\perp} \mathcal{W}$ induces a bijection

$$
\text { Serre }\left(\mathrm{W}_{\mathrm{L}}(\mathcal{T})\right) \rightarrow\{\mathcal{U} \in \text { tors } \mathcal{A} \mid[\mathcal{U}, \mathcal{T}] \text { is a wide interval }\}
$$

As a consequence, we obtain the following characterization of widely generated torsion classes:

Theorem 1.8 (Theorem 7.2 ). For $\mathcal{T} \in$ tors $\mathcal{A}$ the following conditions are equivalent:

(a) $\mathcal{T}$ is a widely generated torsion class.

(b) $\mathcal{T}=\mathrm{T}\left(\mathrm{W}_{\mathrm{L}}(\mathcal{T})\right)$.

(c) $\mathcal{T}=\mathrm{T}(\mathcal{L})$, where $\mathcal{L}$ is the set of labels of Hasse arrows in tors $\mathcal{A}$ starting at $\mathcal{T}$.

(d) For every $\mathcal{U} \in$ tors $\mathcal{A}$ with $\mathcal{U} \subsetneq \mathcal{T}$, there exists a Hasse arrow $\mathcal{T} \rightarrow \mathcal{U}^{\prime}$ such that $\mathcal{U} \subseteq \mathcal{U}^{\prime}$.

\section{Setting And notation}

In this section we recall some fundamental facts about partially ordered sets and abelian length categories and describe the standing assumptions of this paper.

2.1. Partially ordered sets. Let $L=(L, \leq)$ be a partially ordered set.

First, we define the opposite partially ordered set $L^{\mathrm{op}}:=\left(L^{\mathrm{op}}, \leq^{\mathrm{op}}\right)$ of $L$ with the same underlying set $L=L^{\mathrm{op}}$ and the partial order $\leq^{\mathrm{op}}$ such that $y \leq{ }^{\mathrm{op}} x$ holds if and only if $x \leq y$ for all $x, y \in L$.

An isomorphism of partially ordered sets $\Phi:(L, \leq) \rightarrow\left(L^{\prime}, \leq^{\prime}\right)$ is a bijection of sets $\Phi: L \rightarrow L^{\prime}$ such that $\Phi(y) \leq \Phi(x)$ if and only if $y \leq x$.

An interval in $L$ is a subset of the form

$$
[y, x]:=\{z \in L \mid y \leq z \leq x\}
$$

where $y \leq x$ are elements of $L$.

The Hasse quiver Hasse $L$ has $L$ as its set of vertices, and there is an arrow $x \rightarrow y$ for $x, y \in L$ if and only if $y<x$ and $[y, x]=\{y, x\}$.

For an interval $[y, x]$ in $L$, we define its set of upper (resp. lower) elements as follows:

$$
\begin{aligned}
& {[y, x]^{+}:=\{x\} \cup\{z \in[y, x] \mid \text { there exists }(x \rightarrow z) \in \text { Hasse } L\},} \\
& {[y, x]^{-}:=\{y\} \cup\{z \in[y, x] \mid \text { there exists }(z \rightarrow y) \in \text { Hasse } L\} .}
\end{aligned}
$$

In particular, if $y=x$, then $[y, x]^{+}=[y, x]^{-}=\{x\}$.

Let $S \subseteq L$ be a subset. A meet (resp. join) of $S$ is a largest (resp. smallest) element in the set $\{y \in L \mid y \leq x$ for all $x \in S\}$ (resp. $\{y \in L \mid y \geq x$ for all $x \in S\}$ ). If a meet (resp. join) of $S$ exists, then it is necessarily unique, and we will refer to it as the meet (resp. the join) of $S$ denoted by

$$
\bigwedge S=\bigwedge_{x \in S} x \quad\left(\operatorname{resp} . \bigvee S=\bigvee_{x \in S} x\right) .
$$

If a meet and a join of every subset of $L$ exists, we call $L$ a complete lattice. 
2.2. Abelian length categories. In this paper, every category is assumed to be essentially small, and every subcategory is a full subcategory closed under isomorphism classes. Throughout $\mathcal{A}$ always denotes an abelian length category, that is, every object $X \in \mathcal{A}$ has a finite filtration $0=X_{0} \subsetneq X_{1} \subsetneq \cdots \subsetneq X_{l}=X$ where each subfactor $X_{i+1} / X_{i}$ a simple object in $\mathcal{A}$.

The opposite category $\mathcal{A}^{\mathrm{op}}$ of $\mathcal{A}$ is defined to be the category with the same objects as $\mathcal{A}$, but $\operatorname{Hom}_{\mathcal{A}}$ op $(X, Y):=\operatorname{Hom}_{\mathcal{A}}(Y, X)$ for all $X, Y \in \mathcal{A}$. Note that $\mathcal{A}^{\mathrm{op}}$ is again an essentially small abelian length category if $\mathcal{A}$ is so.

For a subcategory $\mathcal{C} \subseteq \mathcal{A}$ define the following subcategories of $\mathcal{A}$ :

add $\mathcal{C}:=\left\{X \in \mathcal{A} \mid\right.$ there exist $C \in \mathcal{C}, n \in \mathbb{N}$ and a split epimorphism $\left.C^{n} \rightarrow X\right\}$,

Filt $\mathcal{C}:=\left\{X \in \mathcal{A} \mid\right.$ there exists $0=X_{0} \subseteq X_{1} \subseteq \cdots \subseteq X_{n}=X$ with $\left.X_{i} / X_{i-1} \in \operatorname{add} \mathcal{C}\right\}$,

Fac $\mathcal{C}:=\{X \in \mathcal{A} \mid$ there exist $C \in \operatorname{add} \mathcal{C}$ and an epimorphism $C \rightarrow X$ in $\mathcal{A}\}$,

$\operatorname{Sub\mathcal {C}}:=\{X \in \mathcal{A} \mid$ there exist $C \in \operatorname{add} \mathcal{C}$ and a monomorphism $X \hookrightarrow C$ in $\mathcal{A}\}$,

$\mathcal{C}^{\perp}:=\left\{X \in \mathcal{A} \mid\right.$ for all $\left.C \in \mathcal{C}, \operatorname{Hom}_{\mathcal{A}}(C, X)=0\right\}$,

${ }^{\perp} \mathcal{C}:=\left\{X \in \mathcal{A} \mid\right.$ for all $\left.C \in \mathcal{C}, \operatorname{Hom}_{\mathcal{A}}(X, C)=0\right\}$.

We will use abbreviation rules such as

$$
\operatorname{add}\left(\mathcal{C}_{1}, \ldots, \mathcal{C}_{m}, X_{1}, \ldots, X_{n}\right):=\operatorname{add}\left(\mathcal{C}_{1} \cup \cdots \cup \mathcal{C}_{m} \cup\left\{X_{1}, \ldots, X_{n}\right\}\right)
$$

for $\mathcal{C}_{1}, \ldots, \mathcal{C}_{m} \subseteq \mathcal{A}$ and $X_{1}, \ldots, X_{n} \in \mathcal{A}$. Also, if $\mathcal{C}, \mathcal{C}^{\prime} \subseteq \mathcal{A}$, then we write $\mathcal{C} * \mathcal{C}^{\prime}$ for the full subcategory of objects $X$ admitting a short exact sequence

$$
0 \rightarrow C \rightarrow X \rightarrow C^{\prime} \rightarrow 0
$$

with $C \in \mathcal{C}$ and $C^{\prime} \in \mathcal{C}^{\prime}$.

A subcategory $\mathcal{W} \subseteq \mathcal{A}$ is called wide if $\mathcal{W}$ is closed under kernels, cokernels and extensions in $\mathcal{A}$. In particular, every wide subcategory of $\mathcal{A}$ is an abelian subcategory. We denote by $\operatorname{sim} \mathcal{W}$ the set of isomorphism classes of simple objects in $\mathcal{W}$. By abuse of notation we will not always distinguish between isomorphism classes in $\operatorname{sim} \mathcal{W}$ and their representatives. Write wide $\mathcal{A}$ for the set of wide subcatgeories of $\mathcal{A}$.

A brick in $\mathcal{A}$ is an object $S \in \mathcal{A}$ such that $\operatorname{End}_{A}(S)$ is a division ring. For a subcategory $\mathcal{C} \subseteq \mathcal{A}$, let brick $\mathcal{C}$ be the set of isomorphism classes of bricks in $\mathcal{C}$. A set $\mathcal{S}$ of isomorphism classes of bricks in $\mathcal{A}$ is called a semibrick if its elements are pairwise Hom-orthogonal, that is, $\operatorname{Hom}_{A}\left(S, S^{\prime}\right)=0$ for any two representatives $S$ and $S^{\prime}$ of distinct isomorphism classes in $\mathcal{S}$. Let sbrick $\mathcal{A}$ be the set of semibricks in $\mathcal{A}$.

A classical result of Ringel [Rin, 1.2] tells us that Filt and sim induce mutually inverse bijections

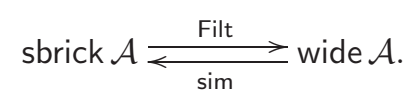

A subcategory $\mathcal{W}$ of $\mathcal{A}$ is called a Serre subcategory if it is closed under extensions, factor objects and subobjects. Since $\mathcal{A}$ is an abelian length category, a subcategory $\mathcal{W} \subseteq \mathcal{A}$ is a Serre subcategory if and only if $\mathcal{W}=$ Filt $\mathcal{S}$ for a subset $\mathcal{S} \subseteq \operatorname{sim} \mathcal{A}$. We denote the set of Serre subcategories of $\mathcal{A}$ by Serre $\mathcal{A}$. This set can be identified with the power set of $\operatorname{sim} \mathcal{A}$.

A torsion pair $(\mathcal{T}, \mathcal{F})$ in $\mathcal{A}$ is a pair of subcategories $\mathcal{T}, \mathcal{F} \subseteq \mathcal{A}$ such that

$$
\mathcal{F}=\mathcal{T}^{\perp} \text { and } \mathcal{T}={ }^{\perp} \mathcal{F} \text {. }
$$

For a torsion pair $(\mathcal{T}, \mathcal{F})$ and every object $X \in \mathcal{A}$, there exists a short exact sequence

$$
0 \rightarrow t_{\mathcal{T}} X \rightarrow X \rightarrow f_{\mathcal{F}} X \rightarrow 0
$$


with $t_{\mathcal{T}} X \in \mathcal{T}$ and $f_{\mathcal{F}} X \in \mathcal{F}$, unique up to isomorphism. We will refer to (2.1) as the canonical sequence of $X$ with respect to the torsion pair $(\mathcal{T}, \mathcal{F})$. Note that the subcategory $\mathcal{T}$ in a torsion pair $(\mathcal{T}, \mathcal{F})$ is closed under extensions and taking factor objects. Dually, the subcategory $\mathcal{F}$ is closed under extensions and subobjects. Conversely, one can show that, given a subcategory $\mathcal{T} \subseteq \mathcal{A}$ closed under extensions and factor objects, the pair $\left(\mathcal{T}, \mathcal{T}^{\perp}\right)$ is a torsion pair, and we call such subcategories $\mathcal{T}$ torsion classes. The set of torsion classes in $\mathcal{A}$ is denoted by tors $\mathcal{A}$. Dually, if $\mathcal{F} \subseteq \mathcal{A}$ is a subcategory closed under extensions and subobjects, then $\left({ }^{\perp} \mathcal{F}, \mathcal{F}\right)$ is a torsion pair, and we call $\mathcal{F}$ a torsion-free class. The set of torsion-free classes in $\mathcal{A}$ is denoted by torf $\mathcal{A}$.

The sets tors $\mathcal{A}$ and torf $\mathcal{A}$ are partially ordered by inclusion and closed under arbitrary intersections. In particular, given a full subcategory $\mathcal{X} \subseteq \mathcal{A}$,

$$
\mathrm{T}(\mathcal{X}):=\bigcap_{\mathcal{T} \in \text { tors } \mathcal{A} ; \mathcal{X} \subseteq \mathcal{T}} \mathcal{T} \text { and } \mathrm{F}(\mathcal{X}):=\bigcap_{\mathcal{F} \in \text { torf } \mathcal{A} ; \mathcal{X} \subseteq \mathcal{F}} \mathcal{F}
$$

are the smallest torsion class and the smallest torsion-free class containing $\mathcal{X}$ respectively. It is well known that $\mathrm{T}=$ Filt $\circ \mathrm{Fac}$ and $\mathrm{F}=$ Filt $\circ$ Sub, see $\mathrm{MS}$, Lemma 3.1] for a proof. These operators show that tors $\mathcal{A}$ and torf $\mathcal{A}$ are complete lattices with joins and meets given by

$$
\begin{array}{ll}
\bigvee S=\bigvee_{\mathcal{T} \in S} \mathcal{T}=\mathrm{T}\left(\bigcup_{\mathcal{T} \in S} \mathcal{T}\right), & \bigwedge S=\bigwedge_{\mathcal{T} \in S} \mathcal{T}=\bigcap_{\mathcal{T} \in S} \mathcal{T}, \\
\bigvee S^{\prime}=\bigvee_{\mathcal{F} \in S^{\prime}} \mathcal{F}=\mathrm{F}\left(\bigcup_{\mathcal{F} \in S^{\prime}} \mathcal{F}\right), & \bigwedge S^{\prime}=\bigwedge_{\mathcal{F} \in S^{\prime}} \mathcal{F}=\bigcap_{\mathcal{F} \in S^{\prime}} \mathcal{F}
\end{array}
$$

for $S \subseteq$ tors $\mathcal{A}$ and $S^{\prime} \subseteq$ torf $\mathcal{A}$. We remark that $\left(\mathrm{T}(\mathcal{X}), \mathcal{X}^{\perp}\right)$ and $\left({ }^{\perp} \mathcal{X}, \mathrm{F}(\mathcal{X})\right)$ are torsion pairs in $\mathcal{A}$ for any full subcategory $\mathcal{X} \subseteq \mathcal{A}$.

In examples, we sometimes let $A$ be a finite-dimensional algebra over a field $K$. Then we let $\mathcal{A}=\bmod A$ be the category of finite-dimensional $A$-modules, and write tors $A:=\operatorname{tors}(\bmod A)$. We write $\operatorname{proj} A$ for the category of finitely generated projective $A$-modules.

\section{BRICK LABELING}

Let $[\mathcal{U}, \mathcal{T}]$ be an interval in tors $\mathcal{A}$. The subcategory $\mathcal{U}^{\perp} \cap \mathcal{T}$ measures the "difference" between $\mathcal{U}$ and $\mathcal{T}$ in the following sense:

Lemma 3.1. Let $[\mathcal{U}, \mathcal{T}]$ be an interval in tors $\mathcal{A}$ and set $\mathcal{W}:=\mathcal{U}^{\perp} \cap \mathcal{T}$. Then for every $T \in \mathcal{T}$ there is a short exact sequence

$$
0 \rightarrow U \rightarrow T \rightarrow W \rightarrow 0
$$

with $U \in \mathcal{U}$ and $W \in \mathcal{W}$, unique up to isomorphism of short exact sequences. Thus $\mathcal{T}=\mathcal{U} * \mathcal{W}$.

Proof. Take the canonical sequence (2.1) for $T$ with respect to the torsion pair $\left(\mathcal{U}, \mathcal{U}^{\perp}\right)$ and let $U:=t_{\mathcal{U}} T \in \mathcal{U}$ and $W:=f_{\mathcal{U}} \perp \in \mathcal{U}^{\perp}$. Since $\mathcal{T}$ is closed under factor objects, we obtain $W \in \mathcal{T}$; hence $W \in \mathcal{U}^{\perp} \cap \mathcal{T}=\mathcal{W}$. Conversely, the desired exact sequence is unique up to isomorphism by the uniqueness of the canonical sequence for $T$. Thus we obtain the first statement, which readily implies that $\mathcal{T}=\mathcal{U} * \mathcal{W}$.

In DIRRT, the following fundamental properties of intervals in tors $\mathcal{A}$ are established.

Proposition 3.2. Let $[\mathcal{U}, \mathcal{T}]$ be an interval in tors $\mathcal{A}$. 
(1) [DIRRT, Lemma 3.10] Then $\mathcal{U}^{\perp} \cap \mathcal{T}=\operatorname{Filt}\left(\operatorname{brick}\left(\mathcal{U}^{\perp} \cap \mathcal{T}\right)\right)$.

(2) [DIRRT, Theorems 3.3,3.4] There is an arrow $q: \mathcal{T} \rightarrow \mathcal{U}$ in Hasse(tors $\mathcal{A})$ if and only if there exists a unique brick $S_{q} \in \operatorname{brick}\left(\mathcal{U}^{\perp} \cap \mathcal{T}\right)$ up to isomorphism. In this case,

$$
\mathcal{U}^{\perp} \cap \mathcal{T}=\text { Filt } S_{q}
$$

and, moreover, $\mathcal{U}=\mathcal{T} \cap{ }^{\perp} S_{q}$ and $\mathcal{T}=\mathrm{T}\left(\mathcal{U}, S_{q}\right)$.

Proposition 3.2 gives rise to the following brick labeling.

Definition 3.3 (DIRRT, Definition 3.5]). The brick label of an arrow $q: \mathcal{T} \rightarrow \mathcal{U}$ in Hasse (tors $\mathcal{A}$ ) is the isomorphism class of the brick $S_{q}$ from Proposition 3.2. In this case, we write $\mathcal{T} \stackrel{S_{q}}{\longrightarrow} \mathcal{U}$.

The brick labeling may be compared to minimal extending modules for $\mathcal{U}$ as defined in Barnard-Carroll-Zhu $\mathrm{BCZ}$. The minimal extending modules for $\mathcal{U}$ are precisely the labels of arrows ending in $\mathcal{U}$, see [BCZ, Subsection 2.2].

Dually, define brick labeling of Hasse $($ torf $\mathcal{A}$ ) so that an arrow $\mathcal{F} \rightarrow \mathcal{G}$ is labeled by the unique brick in ${ }^{\perp} \mathcal{G} \cap \mathcal{F}$. There is the following strong relationship between tors $\mathcal{A}$ and torf $\mathcal{A}$ compatible with their brick labelings.

Proposition 3.4. The construction of right and left Hom-orthogonal subcategories defines isomorphisms of partially ordered sets

$$
\text { (tors } \mathcal{A})^{\mathrm{op}} \stackrel{\bullet^{\perp}}{\stackrel{\bullet}{\rightleftarrows}} \text { torf } \mathcal{A} \text {. }
$$

Moreover, these isomorphisms preserve the brick labeling in the following sense: The brick label of $\mathcal{T} \rightarrow \mathcal{U}$ in Hasse(tors $\mathcal{A}$ ) is the same as the brick label of $\mathcal{U}^{\perp} \rightarrow \mathcal{T}^{\perp}$ in Hasse (torf $\mathcal{A})$.

Proof. The isomorphism (tors $\mathcal{A})^{\mathrm{op}} \cong \operatorname{torf} \mathcal{A}$ is well known and elementary. We show the invariance of the brick labeling. Let $\mathcal{T} \stackrel{S_{q}}{\longrightarrow} \mathcal{U}$ be a labeled arrow in Hasse $($ tors $\mathcal{A})$. Note that ${ }^{\perp}\left(\mathcal{T}^{\perp}\right)=\mathcal{T}$ since $\mathcal{T}$ is a torsion class in $\mathcal{A}$. This basic observation implies

$$
{ }^{\perp}\left(\mathcal{T}^{\perp}\right) \cap \mathcal{U}^{\perp}=\mathcal{U}^{\perp} \cap \mathcal{T}=\text { Filt } S_{q} ;
$$

hence, according to the dual of Definition $3.3 \mathcal{U}^{\perp} \rightarrow \mathcal{T}^{\perp}$ has the label $S_{q}$ in Hasse $($ torf $\mathcal{A})$.

Brick labeling between torsion classes was first considered for the preprojective algebras $\Pi$ of Dynkin type $\Delta$ in IRRT. The crucial ingredient is the bijection between the Coxeter group $W$ associated to $\Delta$ and tors $\Pi$ established by Mizuno Miz. The first named author of this paper introduced brick labeling in Asa1] for functorially finite torsion classes in the module category $\bmod A$ in the context of the Koenig-Yang correspondences [KY, BY] and $\tau$-tilting theory [AIR].

One can easily check that the labeled Hasse quiver of tors $\mathcal{A}$ has the following global structure:

Proposition 3.5. For an abelian length category $\mathcal{A}$, the following properties hold:

- The arrows in Hasse(tors $\mathcal{A})$ ending in 0 are Filt $S \stackrel{S}{\rightarrow} 0$ where $S$ runs through $\operatorname{sim} \mathcal{A}$.

- The arrows in Hasse(tors $\mathcal{A})$ starting at $\mathcal{A}$ are $\mathcal{A} \stackrel{S}{\rightarrow}{ }^{\perp} S$ where $S$ runs through $\operatorname{sim} \mathcal{A}$.

- For $S, S^{\prime} \in \operatorname{sim} \mathcal{A}$, there is an inclusion Filt $S \subseteq{ }^{\perp} S^{\prime}$ if and only if $S \neq S^{\prime}$. 
Proposition 3.7 below shows that the set of labels of arrows in Hasse(tors $\mathcal{A}$ ) starting at (resp. ending in) a fixed torsion class actually form a semibrick. In order to simplify our statements, we introduce the following notation.

Notation 3.6. For a subset $S \subseteq$ tors $\mathcal{A}$, let Label $S$ denote the set of labels of arrows in the full subquiver of Hasse(tors $\mathcal{A})$ with vertices in $S$.

Proposition 3.7. Let $[\mathcal{U}, \mathcal{T}]$ be an interval in tors $\mathcal{A}$. The sets Label $[\mathcal{U}, \mathcal{T}]^{+}$and Label $[\mathcal{U}, \mathcal{T}]^{-}$are semibricks.

Proof. We prove only the first statement; the second one can be shown similarly.

Since all labels are bricks by Definition 3.3, it is enough to show Hom-orthogonality. Let $\mathcal{T}_{i} \stackrel{S_{i}}{\longrightarrow} \mathcal{T}$ be arrows in Hasse(tors $\mathcal{A}$ ) for $i \in\{1,2\}$ with $\mathcal{T}_{1} \neq \mathcal{T}_{2}$. We first remark that $S_{1} \neq S_{2}$ follows from Proposition 3.2

Let $f \in \operatorname{Hom}_{\mathcal{A}}\left(S_{1}, S_{2}\right)$. There is a short exact sequence

$$
0 \rightarrow X^{\prime} \rightarrow \operatorname{Cok} f \rightarrow X^{\prime \prime} \rightarrow 0
$$

with $X^{\prime} \in \mathcal{T}$ and $X^{\prime \prime} \in \mathcal{T}^{\perp}$. Since $X^{\prime \prime}$ is a factor object of $S_{2}$, we deduce that $X^{\prime \prime} \in \mathcal{T}^{\perp} \cap \mathcal{T}_{2}$. Then Proposition 3.2 implies $X^{\prime \prime} \in$ Filt $S_{2}$. Thus $X^{\prime \prime}$ must be 0 or isomorphic to $S_{2}$.

If $X^{\prime \prime}=0$, then Cok $f \in \mathcal{T}$. Thus in the short exact sequence

$$
0 \rightarrow \operatorname{Im} f \rightarrow S_{2} \rightarrow \operatorname{Cok} f \rightarrow 0,
$$

Im $f \in \mathrm{T}\left(S_{1}\right) \subseteq \mathcal{T}_{1}$ and Cok $f \in \mathcal{T} \subseteq \mathcal{T}_{1}$ hold, so $S_{2} \in \mathcal{T}_{1}$. Clearly, $S_{2} \in \mathcal{T}^{\perp}$, so $S_{2} \in \mathcal{T}^{\perp} \cap \mathcal{T}_{1}$. Then Proposition 3.2 gives $S_{2} \cong S_{1}$, but this is a contradiction. Thus $X^{\prime \prime} \cong S_{2}$, and then Cok $f=S_{2}$. This implies $f=0$ as desired.

\section{WidE INTERVALS AND REDUCTION OF TORSION CLASSES}

In this section we investigate wide intervals $[\mathcal{U}, \mathcal{T}]$ in tors $\mathcal{A}$.

Definition 4.1. An interval $[\mathcal{U}, \mathcal{T}]$ in tors $\mathcal{A}$ is a wide interval if $\mathcal{U}^{\perp} \cap \mathcal{T}$ is a wide subcategory of $\mathcal{A}$.

For example, we can construct a wide interval $[\mathcal{U}(N, Q), \mathcal{T}(N, Q)] \subseteq$ tors $A$ from a $\tau$-rigid pair $(N, Q)$ in $\bmod A$ (that is, $N \in \bmod A$ and $Q \in \operatorname{proj} A$ satisfying $\operatorname{Hom}_{A}(N, \tau N)=0$ and $\left.\operatorname{Hom}_{A}(Q, N)=0\right)$, where

$$
\mathcal{U}(N, Q):=\operatorname{Fac} N \text { and } \mathcal{T}(N, Q):=^{\perp}(\tau N) \cap Q^{\perp}
$$

as in [Jas, DIRRT]. In this case, $[\mathcal{U}(N, Q), \mathcal{T}(N, Q)]$ is isomorphic to tors $C_{N, Q}$ for a certain finite-dimensional $K$-algebra $C_{N, Q}$ [DIRRT, Theorem 4.12] (see also [Jas, Theorems $3.8,3.12]$ ) as a complete lattice. This bijection is compatible with the brick labeling of $[\mathcal{U}(N, Q), \mathcal{T}(N, Q)] \subseteq$ tors $A$ and tors $C_{N, Q}$ DIRRT, Proposition 4.13]. We extend these results for all wide intervals as follows.

Theorem 4.2. Let $[\mathcal{U}, \mathcal{T}]$ be a wide interval in tors $\mathcal{A}$, and set $\mathcal{W}:=\mathcal{U}^{\perp} \cap \mathcal{T}$.

(1) There are mutually inverse isomorphisms of complete lattices

$$
[\mathcal{U}, \mathcal{T}] \underset{\Psi}{\stackrel{\Phi}{\rightleftarrows}} \operatorname{tors} \mathcal{W}
$$

given by $\Phi(\mathcal{V}):=\mathcal{U}^{\perp} \cap \mathcal{V}$ and $\Psi(\mathcal{X}):=\mathrm{T}(\mathcal{U}, \mathcal{X})$ for $\mathcal{V} \in[\mathcal{U}, \mathcal{T}]$ and $\mathcal{X} \in$ tors $\mathcal{W}$ respectively. Moreover, $\Psi(\mathcal{X})=\mathcal{U} * \mathcal{X}$ holds for every $\mathcal{X} \in$ tors $\mathcal{W}$.

(2) The isomorphism $\Phi$ preserves the brick labeling: Namely, the brick label of $\mathcal{V}_{1} \rightarrow \mathcal{V}_{2}$ in $[\mathcal{U}, \mathcal{T}]$ is the same as the brick label of $\Phi\left(\mathcal{V}_{1}\right) \rightarrow \Phi\left(\mathcal{V}_{2}\right)$ in tors $\mathcal{W}$.

(3) We have $\operatorname{sim} \mathcal{W}=$ Label $[\mathcal{U}, \mathcal{T}]^{+}=$Label $[\mathcal{U}, \mathcal{T}]^{-}$. 
Proof. (1) Both maps are easily seen to be well defined morphisms of partially ordered sets.

For $\mathcal{V} \in[\mathcal{U}, \mathcal{T}]$, the identity $\mathcal{V}=\mathcal{U} *\left(\mathcal{U}^{\perp} \cap \mathcal{V}\right)$ follows immediately from Lemma 3.1, but $\mathcal{U} *\left(\mathcal{U}^{\perp} \cap \mathcal{V}\right) \subseteq \mathrm{T}\left(\mathcal{U}, \mathcal{U}^{\perp} \cap \mathcal{V}\right)$ hence $\mathcal{V}=\mathrm{T}\left(\mathcal{U}, \mathcal{U}^{\perp} \cap \mathcal{V}\right)$ by minimality. This proves $\Psi \circ \Phi=$ id.

Let $\mathcal{X} \in$ tors $\mathcal{W}$. Since $\mathcal{X} \subseteq \mathcal{W} \subseteq \mathcal{U}^{\perp}$, the inclusion $\mathcal{X} \subseteq \mathcal{U}^{\perp} \cap \mathrm{T}(\mathcal{U}, \mathcal{X})$ is obvious. For the other one, let $Y \in \mathcal{U}^{\perp} \cap \mathrm{T}(\mathcal{U}, \mathcal{X})$. We use induction on the length of $Y$ in $\mathcal{W}$. If $Y=0$, then the claim is clear. Assume $Y \neq 0$; then there is a short exact sequence

$$
0 \rightarrow Y^{\prime} \rightarrow Y \rightarrow Y^{\prime \prime} \rightarrow 0
$$

with $0 \neq Y^{\prime} \in \mathrm{T}(\mathcal{X}) \subseteq \mathcal{A}$ and $Y^{\prime \prime} \in \mathcal{X}^{\perp}$. Then $Y^{\prime} \in \mathcal{U}^{\perp}$ because $Y^{\prime}$ is a subobject of $Y$, and $Y^{\prime} \in \mathrm{T}(\mathcal{X}) \subseteq \mathcal{T}$ follows from $\mathcal{X} \subseteq \mathcal{W} \subseteq \mathcal{T}$. Thus $Y^{\prime} \in \mathcal{U}^{\perp} \cap \mathcal{T} \cap \mathrm{T}(\mathcal{X})=$ $\mathcal{W} \cap \mathrm{T}(\mathcal{X})=\mathcal{X}$, since $\mathcal{X} \in$ tors $\mathcal{W}$. Moreover $\bar{Y}^{\prime \prime} \cong \operatorname{Cok}\left(Y^{\prime} \rightarrow Y\right) \in \mathcal{W}$ as $\mathcal{W}$ is assumed to be wide, and we have $Y^{\prime \prime} \in \mathcal{U}^{\perp} \cap \mathrm{T}(\mathcal{U}, \mathcal{X})$ since $\mathcal{W} \subseteq \mathcal{U}^{\perp}$ and $Y^{\prime \prime}$ is a factor object of $Y \in \mathrm{T}(\mathcal{U}, \mathcal{X})$. Now we may apply induction on the length to $Y^{\prime \prime}$. We obtain $Y^{\prime \prime} \in \mathcal{X}$ and henceforth $Y \in \mathcal{X}$. This proves $\Phi \circ \Psi=$ id.

Altogether $\Phi$ and $\Psi$ are mutually inverse isomorphisms of partially ordered sets thus also isomorphisms of complete lattices.

For the last statement, let $\mathcal{X} \in$ tors $\mathcal{W}$, then we get

$$
\Psi(\mathcal{X})=\mathcal{U} *\left(\mathcal{U}^{\perp} \cap \Psi(\mathcal{X})\right)=\mathcal{U} * \Phi(\Psi(\mathcal{X}))=\mathcal{U} * \mathcal{X}
$$

(2) For every interval $\left[\mathcal{V}_{1}, \mathcal{V}_{2}\right]$ in $[\mathcal{U}, \mathcal{T}]$ the equality

$$
\left(\mathcal{U}^{\perp} \cap \mathcal{V}_{1}\right)^{\perp} \cap\left(\mathcal{U}^{\perp} \cap \mathcal{V}_{2}\right)=\mathcal{V}_{1}^{\perp} \cap \mathcal{V}_{2} \subseteq \mathcal{W}
$$

holds, hence $\Phi$ preserves the brick labeling by Proposition 3.2 , and so does $\Psi$.

(3) In view of Proposition 3.5. this follows from parts (1) and (2).

Let us give an example of a wide interval which does not come from $\tau$-rigid pairs.

Example 4.3. Assume that $K$ is an algebraically closed field and that $A$ is the path algebra $K(1 \rightrightarrows 2)$ of the Kronecker quiver $1 \rightrightarrows 2$.

We set $\mathcal{T}$ as the smallest torsion class containing all regular and preinjective modules, and $\mathcal{U}$ as the smallest torsion class containing all preinjective modules.

In this case, $\mathcal{W}$ is a wide subcategory of $\bmod A$, namely the subcategory of all regular $A$-modules. Thus $[\mathcal{U}, \mathcal{T}]$ is a wide interval. The simple objects in $\mathcal{W}$ are all quasi-simple regular modules $S_{\lambda}$ parametrized by $\lambda \in \mathbb{P}^{1}(K)$. One can check that $\mathcal{W} \simeq \bigoplus_{\lambda \in \mathbb{P}^{1}(K)}$ Filt $S_{\lambda}$ as an abelian category, so tors $\mathcal{W}$ can be identified with $\prod_{\lambda \in \mathbb{P}^{1}(K)}$ tors $\left(\right.$ Filt $\left.S_{\lambda}\right)$. Since the torsion classes in Filt $S_{\lambda}$ are Filt $S_{\lambda}$ and $\{0\}$, tors $\mathcal{W}$ is in bijection with the power set $2^{\mathbb{P}^{1}(K)}$.

Therefore Theorem 4.2 gives an isomorphism of complete lattices

$$
2^{\mathbb{P}^{1}(K)} \rightarrow[\mathcal{U}, \mathcal{T}] ; \quad \Lambda \mapsto \mathcal{U} * \operatorname{Filt}\left\{S_{\lambda} \mid \lambda \in \Lambda\right\}
$$

Every arrow ending in the smallest element $\{0\} \in$ tors $\mathcal{W}$ is of the form Filt $S_{\lambda} \rightarrow\{0\}$ for some $\lambda \in \mathbb{P}^{1}(K)$, and it is labeled by the brick $S_{\lambda}$. The corresponding arrow in $[\mathcal{U}, \mathcal{T}]$ is $\mathrm{T}\left(\mathcal{U}, S_{\lambda}\right) \rightarrow \mathcal{U}$, and its brick label is also $S_{\lambda}$.

We remark that this example can be obtained also from numerical torsion classes

$$
\begin{aligned}
\mathcal{T}_{\theta} & :=\{M \in \bmod A \mid \text { for any nonzero quotient } N, \theta(N)>0\}, \\
\overline{\mathcal{T}}_{\theta} & :=\{M \in \bmod A \mid \text { for any quotient } N, \theta(N) \geq 0\} .
\end{aligned}
$$

associated to each $\theta \in K_{0}(\operatorname{proj} A) \otimes_{\mathbb{Z}} \mathbb{R}$ in BKT, Bri2]. For any $\theta$, the intersection $\mathcal{T}_{\theta}^{\perp} \cap \overline{\mathcal{T}}_{\theta}$ is the $\theta$-semistable subcategory

$\mathcal{W}_{\theta}:=\{M \in \bmod A \mid \theta(M)=0$, and for any nonzero quotient $N, \theta(N) \geq 0\}$ 
introduced by King $\underline{\mathrm{Kin}}$, which is a wide subcategory. By setting $\theta:=\left[P_{1}\right]-\left[P_{2}\right]$ with $P_{i}$ the indecomposable projective module, we get $\mathcal{T}_{\theta}=\mathcal{U}$ and $\overline{\mathcal{T}}_{\theta}=\mathcal{T}$ above. Thus, $[\mathcal{U}, \mathcal{T}]$ is a wide interval, and the simple objects of $\mathcal{W}_{\theta}$ are $S_{\lambda}$.

Similar arguments hold for tame hereditary algebras.

\section{Classification of Wide intervals in terms of JOIN and meet INTERVALS}

Motivated by the results of Section 4 we next aim for characterizing wide intervals in terms of arrows in the Hasse quiver of torsion classes. For this purpose, we define the following notions for intervals.

Definition 5.1. Let $[\mathcal{U}, \mathcal{T}]$ be an interval in tors $\mathcal{A}$.

(1) The interval $[\mathcal{U}, \mathcal{T}]$ is called a join interval if $\mathcal{T}=\bigvee[\mathcal{U}, \mathcal{T}]^{-}$.

(2) The interval $[\mathcal{U}, \mathcal{T}]$ is called a meet interval if $\mathcal{U}=\bigwedge[\mathcal{U}, \mathcal{T}]^{+}$.

We remark that $[\mathcal{T}, \mathcal{T}]$ is a join interval and a meet interval because $[\mathcal{T}, \mathcal{T}]^{-}=$ $[\mathcal{T}, \mathcal{T}]^{+}=\{\mathcal{T}\}$. Actually, these notions coincide with wide intervals.

Theorem 5.2. Let $[\mathcal{U}, \mathcal{T}]$ be an interval in tors $\mathcal{A}$. Then the following conditions are equivalent:

(a) The interval $[\mathcal{U}, \mathcal{T}]$ is a wide interval.

(b) The interval $[\mathcal{U}, \mathcal{T}]$ is a join interval.

(c) The interval $[\mathcal{U}, \mathcal{T}]$ is a meet interval.

The isomorphisms between the complete lattices in Theorem 4.2 together with Proposition 3.5 imply $(\mathrm{a}) \Rightarrow(\mathrm{b})$ and $(\mathrm{a}) \Rightarrow(\mathrm{c})$. For the remaining parts, we need the following property of join intervals.

Proposition 5.3. Let $[\mathcal{U}, \mathcal{T}]$ be a join interval in tors $\mathcal{A}$, set $\mathcal{W}:=\mathcal{U}^{\perp} \cap \mathcal{T}$ and $\mathcal{L}:=$ Label $[\mathcal{U}, \mathcal{T}]^{-}$. Then $\mathcal{W}=$ Filt $\mathcal{L}$.

Proof. The inclusion Filt $\mathcal{L} \subseteq \mathcal{W}$ is immediate from $\mathcal{W}$ being closed under extensions and containing $\mathcal{L}$. It remains to show the other inclusion.

Proposition 3.2 implies that taking the label $S_{q} \in \mathcal{L}$ of the arrow $q: \mathcal{V} \rightarrow \mathcal{U}$ for $\mathcal{V} \in[\mathcal{U}, \mathcal{T}]^{-} \backslash\{\mathcal{U}\}$ gives a bijection $[\mathcal{U}, \mathcal{T}]^{-} \backslash\{\mathcal{U}\} \rightarrow \mathcal{L}$. Since $[\mathcal{U}, \mathcal{T}]$ is assumed to be a join interval,

$$
\mathcal{T}=\mathcal{U} \vee\left(\bigvee_{S \in \mathcal{L}} \mathrm{T}(\mathcal{U}, S)\right)=\mathrm{T}(\mathcal{U}, \mathcal{L})=\operatorname{Filt}(\operatorname{Fac}(\mathcal{U}, \mathcal{L}))
$$

We first prove that $\mathcal{T}=\operatorname{Filt}(\mathcal{U}, \mathcal{L})$. It is sufficient to show $X \in \operatorname{Filt}(\mathcal{U}, \mathcal{L})$ if $X$ is a factor object of $S \in \mathcal{L}$. Set $\mathcal{U}^{\prime}:=\mathrm{T}(\mathcal{U}, S)$. We can take a short exact sequence $0 \rightarrow X^{\prime} \rightarrow X \rightarrow X^{\prime \prime} \rightarrow 0$ with $X^{\prime} \in \mathcal{U}$ and $X^{\prime \prime} \in \mathcal{U}^{\perp}$. Then $X^{\prime \prime} \in \mathcal{U}^{\perp} \cap \mathcal{U}^{\prime}$, so $X^{\prime \prime} \in$ Filt $S$ by Proposition 3.2 . Thus $X \in \operatorname{Filt}(\mathcal{U}, \mathcal{L})$, so $\mathcal{T}=\operatorname{Filt}(\mathcal{U}, \mathcal{L})$.

To finish the proof, assume $T \in \mathcal{W}$ and show $T \in$ Filt $\mathcal{L}$ by induction on the length of $T$ in $\mathcal{W}$. If $T=0$, it is clear. If $T \neq 0$, then we have a short exact sequence $0 \rightarrow S \rightarrow T \rightarrow T^{\prime} \rightarrow 0$ with $S \in \mathcal{L}$, since $T \in \mathcal{T}=\operatorname{Filt}(\mathcal{U}, \mathcal{L})$ and $T \in \mathcal{W} \subseteq \mathcal{U}^{\perp}$. We obtain the following commutative diagram with exact rows and 
columns:

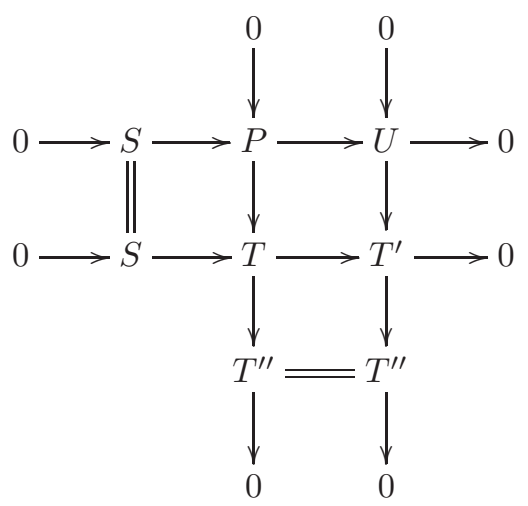

where $U \in \mathcal{U}$ and $T^{\prime \prime} \in \mathcal{U}^{\perp}$. The column $0 \rightarrow U \rightarrow T^{\prime} \rightarrow T^{\prime \prime} \rightarrow 0$ is the canonical sequence of $T^{\prime}$ with respect to $\left(\mathcal{U}, \mathcal{U}^{\perp}\right)$, and the row $0 \rightarrow S \rightarrow P \rightarrow U \rightarrow 0$ is obtained via pullback. Note that $T^{\prime \prime} \in \mathcal{T}$ as a proper factor of $T$, hence we can apply the induction hypothesis to $T^{\prime \prime} \in \mathcal{U}^{\perp} \cap \mathcal{T}=\mathcal{W}$ to obtain $T^{\prime \prime} \in$ Filt $\mathcal{L}$. Moreover, $P \in \mathcal{U}^{\perp}$ as a subobject of $T$ and $P \in \operatorname{Filt}(\mathcal{U}, S)$. Thus $P \in$ Filt $S$ by Proposition 3.2. Therefore $T \in$ Filt $\mathcal{L}$ as an extension of $T^{\prime \prime}$ by $P$.

Now we can complete the proof of Theorem 5.2 .

Proof of Theorem 5.2. Set $\mathcal{W}:=\mathcal{U}^{\perp} \cap \mathcal{T}$ as in the statement of the theorem.

(a) $\Rightarrow$ (b), (a) $\Rightarrow$ (c): These follow from Theorem 4.2 .

(b) $\Rightarrow$ (a): Define $\mathcal{L}:=$ Label $[\mathcal{U}, \mathcal{T}]^{-}$. Proposition 5.3 says that $\mathcal{W}=$ Filt $\mathcal{L}$, and $\mathcal{L}$ is a semibrick by Proposition 3.7. Therefore $\mathcal{W}$ is a wide subcategory of $\mathcal{A}$ (see [Rin, 1.2]).

(c) $\Rightarrow$ (a): Define $\mathcal{L}:=$ Label $[\mathcal{U}, \mathcal{T}]^{+}$. If $[\mathcal{U}, \mathcal{T}]$ is a meet interval, then $\left[\mathcal{T}^{\perp}, \mathcal{U}^{\perp}\right]$ is a join interval in torf $\mathcal{A}$, and Label $\left[\mathcal{T}^{\perp}, \mathcal{U}^{\perp}\right]^{-}=\operatorname{Label}[\mathcal{U}, \mathcal{T}]^{+}=\mathcal{L}$ is a semibrick by Proposition 3.7. Thus, similarly to Proposition 5.3, we have

$$
{ }^{\perp}\left(\mathcal{T}^{\perp}\right) \cap \mathcal{U}^{\perp}=\text { Filt } \mathcal{L} \in \text { wide } \mathcal{A} \text {. }
$$

Therefore $\mathcal{W}=\mathcal{U}^{\perp} \cap \mathcal{T}={ }^{\perp}\left(\mathcal{T}^{\perp}\right) \cap \mathcal{U}^{\perp} \in$ wide $\mathcal{A}$.

We end this section by giving an example illustrating Theorem 5.2 .

Example 5.4. We keep the setting of Example 4.3. Note

$$
[\mathcal{U}, \mathcal{T}]^{-}=\left\{\mathrm{T}\left(\mathcal{U}, S_{\lambda}\right) \mid \lambda \in \mathbb{P}^{1}(K)\right\} .
$$

One can check that $\mathcal{T}$ coincides with the join $\bigvee_{\lambda \in \mathbb{P}^{1}(K)} \mathrm{T}\left(\mathcal{U}, S_{\lambda}\right)$, which means that $[\mathcal{U}, \mathcal{T}]$ is a join interval, and also a wide interval by Theorem 5.2 .

\section{Classification of Wide intervals in terms of Ingalls-Thomas CORRESPONDENCES}

In [IT] Ingalls-Thomas associate to every $\mathcal{T} \in$ tors $\mathcal{A}$ the so called left wide subcategory

$$
\mathrm{W}_{\mathrm{L}}(\mathcal{T}):=\{X \in \mathcal{T} \mid \text { for all } Y \in \mathcal{T} \text { and } g: Y \rightarrow X, \operatorname{Ker} g \in \mathcal{T}\}
$$

in the case that $\mathcal{A}=\bmod A$ for a hereditary algebra $A$; Marks-Š́tovíček studied $\mathrm{W}_{\mathrm{L}}(\mathcal{T})$ in $\mathrm{MS}$ for arbitrary finite-dimensional algebras. Dually to the left wide subcategories associated to torsion classes, one can define the right wide subcategory associated to $\mathcal{F} \in$ torf $\mathcal{A}$ as

$$
\mathrm{W}_{\mathrm{R}}(\mathcal{F}):=\{X \in \mathcal{F} \mid \text { for all } X \in \mathcal{F} \text { and } f: X \rightarrow Y \text {, Cok } f \in \mathcal{F}\} \text {. }
$$


Their following result implies that there exists an injection from wide $\mathcal{A}$ to tors $\mathcal{A}$. We remark that the proof in [MŠ] also works for abelian length categories.

Proposition 6.1 ([IT, Proposition 2.14], [MS̆, Proposition 3.3]). The maps

$$
\text { wide } \mathcal{A} \underset{\mathrm{W}_{\mathrm{L}}}{\stackrel{\mathrm{T}}{\rightleftarrows}} \text { tors } \mathcal{A}
$$

satisfy $\mathrm{W}_{\mathrm{L}} \circ \mathrm{T}=\mathrm{id}$.

In general, $\mathrm{T}:$ wide $\mathcal{A} \rightarrow$ tors $\mathcal{A}$ is not surjective, so it is important to determine the image of the map $\mathrm{T}$. We will answer this problem in the next subsection, and for this purpose, we study the relationship between $\mathcal{T} \in$ tors $\mathcal{A}$ and the left wide subcategory $\mathrm{W}_{\mathrm{L}}(\mathcal{T})$ in this section.

First, we prepare the following property on Serre subcategories of $\mathrm{W}_{\mathrm{L}}(\mathcal{T})$.

Lemma 6.2. Let $\mathcal{T} \in$ tors $\mathcal{A}, \mathcal{W} \in \operatorname{Serre}\left(\mathrm{W}_{\mathrm{L}}(\mathcal{T})\right)$ and $f: X \rightarrow Y$ a homomorphism with $X \in \mathcal{T}$ and $Y \in \mathrm{F}(\mathcal{W})$. Then $\operatorname{Im} f \in \mathcal{W}$ and $\operatorname{Ker} f \in \mathcal{T}$.

Proof. We may assume that $f$ is surjective by replacing $Y$ by $\operatorname{Im} f$. We proceed by induction on the length of $Y$.

If $Y=0$, then the claim is obvious, so assume $Y \neq 0$. Since $Y \in \mathrm{F}(\mathcal{W})=$ Filt $(\operatorname{Sub} \mathcal{W})$, there exists a short exact sequence

$$
0 \rightarrow Y^{\prime \prime} \rightarrow Y \rightarrow Y^{\prime} \rightarrow 0
$$

with $Y^{\prime} \in \operatorname{Sub} \mathcal{W}$ non-zero and $Y^{\prime \prime} \in \mathrm{F}(\mathcal{W})$. We first show $Y^{\prime} \in \mathcal{W}$. Since $Y^{\prime} \in$ Sub $\mathcal{W}, Y^{\prime}$ is a subobject of some $W \in \mathcal{W} \subseteq \mathrm{W}_{\mathrm{L}}(\mathcal{T})$. On the other hand we find $Y^{\prime} \in \mathcal{T}$, because $Y^{\prime}$ is a factor object of $Y \in \mathcal{T}$. These two statements imply that $Y^{\prime} \in \mathrm{W}_{\mathrm{L}}(\mathcal{T})$ by checking the definition of $\mathrm{W}_{\mathrm{L}}(\mathcal{T})$. In the wide subcategory $\mathrm{W}_{\mathrm{L}}(\mathcal{T})$, $Y^{\prime}$ is a subobject of some $W \in \mathcal{W}$, so we obtain $Y^{\prime} \in \mathcal{W}$, since $\mathcal{W} \in \operatorname{Serre}\left(\mathrm{W}_{\mathrm{L}}(\mathcal{T})\right)$.

Next we prove $Y^{\prime \prime} \in \mathcal{W}$. Consider the following commutative diagram with exact rows:

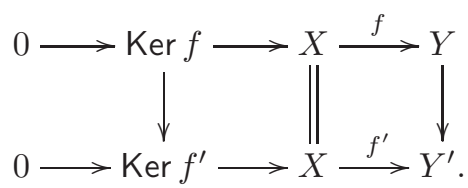

Again, the definition of $\mathrm{W}_{\mathrm{L}}(\mathcal{T})$ yields $\operatorname{Ker} f^{\prime} \in \mathcal{T}$. Since $Y^{\prime \prime}$ is a subobject of $Y \in \mathrm{F}(\mathcal{W})$, we have $Y^{\prime \prime} \in \mathrm{F}(\mathcal{W})$. Now

$$
0 \rightarrow \operatorname{Ker} f \rightarrow \operatorname{Ker} f^{\prime} \rightarrow Y^{\prime \prime} \rightarrow 0
$$

is exact and, by applying the induction hypothesis to $\operatorname{Ker} f^{\prime} \rightarrow Y^{\prime \prime}$, by induction we conclude that $Y^{\prime \prime} \in \mathcal{W}$. Therefore $Y \in \mathcal{W}$ as an extension of $Y^{\prime \prime}$ by $Y^{\prime}$. Now Ker $f \in \mathcal{T}$ follows from the definition of $\mathrm{W}_{\mathrm{L}}(\mathcal{T})$.

We can generalize Proposition 3.2 as follows. This is a mutation of a torsion class $\mathcal{T}$ at a Serre subcategory of $\mathrm{W}_{\mathrm{L}}(\mathcal{T})$.

Proposition 6.3. Let $\mathcal{T} \in$ tors $\mathcal{A}, \mathcal{W} \in \operatorname{Serre}\left(\mathrm{W}_{\mathrm{L}}(\mathcal{T})\right)$ and set $\mathcal{U}:=\mathcal{T} \cap{ }^{\perp} \mathcal{W} \in$ tors $\mathcal{A}$. Then $\mathcal{T}=\mathcal{U} * \mathcal{W}$ and $\mathcal{W}=\mathcal{U}^{\perp} \cap \mathcal{T}$.

Proof. The inclusion $\mathcal{U} * \mathcal{W} \subseteq \mathcal{T}$ is obvious from $\mathcal{U}, \mathcal{W} \subseteq \mathcal{T}$.

For the other inclusion, let $T \in \mathcal{T}$ and take the canonical sequence

$$
0 \rightarrow X \rightarrow T \rightarrow Y \rightarrow 0
$$

with $X \in{ }^{\perp} \mathcal{W}$ and $Y \in \mathrm{F}(\mathcal{W})$. Lemma 6.2 implies $Y \in \mathcal{W}$ and $X \in \mathcal{T} \cap{ }^{\perp} \mathcal{W}=\mathcal{U}$, hence $T \in \mathcal{U} * \mathcal{W}$. 
Next we show $\mathcal{W}=\mathcal{U}^{\perp} \cap \mathcal{T}$. The inclusion $\mathcal{W} \subseteq \mathcal{U}^{\perp} \cap \mathcal{T}$ is easy to check. The other inclusion $\mathcal{U}^{\perp} \cap \mathcal{T} \subseteq \mathcal{W}$ follows from $\mathcal{T}=\mathcal{U} * \mathcal{W}$.

We also need the following technical lemma, which is a generalization of DIRRT, Lemma 3.7] and Asa1, Lemma 2.7].

Lemma 6.4. Let $\mathcal{U} \in$ tors $\mathcal{A}$ and $S \in \mathcal{U}^{\perp}$ a brick. Set $\mathcal{T}:=\mathrm{T}(\mathcal{U}, S)$.

(1) Every homomorphism $f: X \rightarrow S$ in $\mathcal{T}$ is zero or epic and satisfies $\operatorname{Ker} f \in$ $\mathcal{T}$.

(2) The brick $S$ belongs to $\operatorname{sim}\left(\mathrm{W}_{\mathrm{L}}(\mathcal{T})\right)$.

Proof. (1) We use induction on the length of $X$ in $\mathcal{A}$. If $X=0$ the claim is clear, so we assume $X \neq 0$. Since $X \in \mathrm{T}(\mathcal{U}, S)=\mathrm{Filt}(\mathcal{U}$, Fac $S)$, we can take an exact sequence $0 \rightarrow Y \stackrel{a}{\rightarrow} X \rightarrow X^{\prime} \rightarrow 0$ such that $Y$ is non-zero and belongs to $\mathcal{U}$ or Fac $S$. Then $Y \in \mathcal{T}$ in both cases.

If $f a: Y \rightarrow S$ is non-zero, then $Y \in$ Fac $S$ holds, since $S \in \mathcal{U}^{\perp}$. There exists an epimorphism $g: S^{n} \rightarrow Y$, and the composite fag: $S^{n} \rightarrow S$ is non-zero, and hence a split epimorphism of $S$. Thus $f$ is also a split epimorphism and $\operatorname{Ker} f \in \mathcal{T}$ as desired.

On the other hand, if $f a: Y \rightarrow S$ is zero, then $Y \subseteq \operatorname{Ker} f$, so we have a commutative diagram of exact sequences

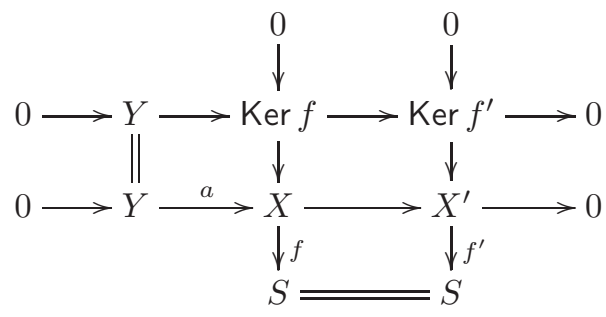

By the induction hypothesis, $f^{\prime}$ is either zero or epic, and satisfies $\operatorname{Ker} f^{\prime} \in \mathcal{T}$. Thus $f$ is either zero or epic, and satisfies $\operatorname{Ker} f \in \mathcal{T}$.

(2) This follows immediately from (1).

Thus $\operatorname{sim}\left(\mathrm{W}_{\mathrm{L}}(\mathcal{T})\right)$ is given by the brick labeling of Hasse(tors $\left.\mathcal{A}\right)$.

Proposition 6.5. Let $\mathcal{T} \in$ tors $\mathcal{A}$ and set $\mathcal{V}:=\mathcal{T} \cap{ }^{\perp} \mathrm{W}_{\mathrm{L}}(\mathcal{T}) \in$ tors $\mathcal{A}$. Then we have $\operatorname{sim}\left(\mathrm{W}_{\mathrm{L}}(\mathcal{T})\right)=$ Label $[\mathcal{V}, \mathcal{T}]^{+}=$Label $[0, \mathcal{T}]^{+}$.

Proof. First $\operatorname{sim}\left(\mathrm{W}_{\mathrm{L}}(\mathcal{T})\right)=$ Label $[\mathcal{V}, \mathcal{T}]^{+}$follows from Proposition 6.3 and Theorem 5.2, and Label $[\mathcal{V}, \mathcal{T}]^{+} \subseteq$ Label $[0, \mathcal{T}]^{+}$is obvious.

Now it remains to show Label $[0, \mathcal{T}]^{+} \subseteq \operatorname{sim}\left(\mathrm{W}_{\mathrm{L}}(\mathcal{T})\right)$. Let $S \in$ Label $[0, \mathcal{T}]^{+}$. Then there exists a Hasse arrow $\mathcal{T} \stackrel{S}{\rightarrow} \mathcal{U}$ in tors $\mathcal{A}$, and Proposition 3.2 implies $\mathcal{T}=\mathrm{T}(\mathcal{U}, S)$; hence $S \in \mathrm{W}_{\mathrm{L}}(\mathcal{T})$ by Lemma 6.4

Now we get a characterization of wide intervals in terms of left and right wide subcategories.

Theorem 6.6. Let $[\mathcal{U}, \mathcal{T}]$ be an interval in tors $\mathcal{A}$ and set $\mathcal{W}:=\mathcal{U}^{\perp} \cap \mathcal{T}$. Then the following conditions are equivalent:
(a) $\mathcal{W} \in$ wide $\mathcal{A}$,
(b) $\mathcal{W} \in \operatorname{Serre}\left(\mathrm{W}_{\mathrm{L}}(\mathcal{T})\right)$,
(c) $\mathcal{W} \in \operatorname{Serre}\left(\mathrm{W}_{\mathrm{R}}\left(\mathcal{U}^{\perp}\right)\right)$,
(d) $\mathcal{W}=\mathrm{W}_{\mathrm{R}}\left(\mathcal{U}^{\perp}\right) \cap \mathrm{W}_{\mathrm{L}}(\mathcal{T})$.

Proof. $(\mathrm{a}) \Rightarrow(\mathrm{b})$ : By assumption $[\mathcal{U}, \mathcal{T}]$ is a wide interval, so $\mathcal{W}=\operatorname{Filt}\left(\right.$ Label $\left.[\mathcal{U}, \mathcal{T}]^{+}\right)$ follows from Theorem 4.2 Therefore $\mathcal{W} \in \operatorname{Serre}\left(\mathrm{W}_{\mathrm{L}}(\mathcal{T})\right)$ according to Proposition 6.5 
(b) $\Rightarrow(a)$ : This is obvious, since by definition Serre subcategories are closed under extensions.

(a) $\Leftrightarrow(\mathrm{c})$ : This follows by duality from (a) $\Leftrightarrow(\mathrm{b})$.

Thus it suffices to show $((\mathrm{b})$ and $(\mathrm{c})) \Leftrightarrow(\mathrm{d})$.

((b) and (c)) $\Rightarrow\left(\right.$ d): This is deduced as $\mathcal{W} \subseteq \mathrm{W}_{\mathrm{R}}\left(\mathcal{U}^{\perp}\right) \cap \mathrm{W}_{\mathrm{L}}(\mathcal{T}) \subseteq \mathcal{U}^{\perp} \cap \mathcal{T}=\mathcal{W}$.

$(\mathrm{d}) \Rightarrow(\mathrm{b})$ : Assume $\mathcal{W}=\mathrm{W}_{\mathrm{R}}\left(\mathcal{U}^{\perp}\right) \cap \mathrm{W}_{\mathrm{L}}(\mathcal{T})$. Since $\mathcal{W}=\mathrm{W}_{\mathrm{R}}\left(\mathcal{U}^{\perp}\right) \cap \mathrm{W}_{\mathrm{L}}(\mathcal{T}) \in$ wide $\left(\mathrm{W}_{\mathrm{L}}(\mathcal{T})\right)$, it remains to check that $\mathcal{W}$ is closed under taking subobjects in $\mathrm{W}_{\mathrm{L}}(\mathcal{T})$. Let $X \in \mathcal{W}$ and $X^{\prime} \subseteq X$ satisfy $X^{\prime} \in \mathrm{W}_{\mathrm{L}}(\mathcal{T})$. As $X \in \mathcal{W} \subseteq \mathcal{U}^{\perp}$, we get $X^{\prime} \in \mathcal{U}^{\perp}$. By assumption, $X^{\prime} \in \mathcal{T}$, so $X^{\prime} \in \mathcal{U}^{\perp} \cap \mathcal{T}=\mathcal{W}$ as desired. Thus $\mathcal{W} \in \operatorname{Serre}\left(\mathrm{W}_{\mathrm{L}}(\mathcal{T})\right)$.

$(\mathrm{d}) \Rightarrow(\mathrm{c})$ : This can be checked as $(\mathrm{d}) \Rightarrow(\mathrm{b})$.

Propositions 6.3, 6.5 and Theorem 6.6 yield the following property:

Theorem 6.7. Let $\mathcal{T} \in$ tors $\mathcal{A}$. Taking labels gives a bijection

$\{$ Hasse arrows in tors $\mathcal{A}$ starting at $\mathcal{T}\} \rightarrow \operatorname{sim}\left(\mathrm{W}_{\mathrm{L}}(\mathcal{T})\right)$.

Moreover, the map $\mathcal{W} \mapsto \mathcal{T} \cap{ }^{\perp} \mathcal{W}$ induces a bijection

$$
\text { Serre }\left(\mathrm{W}_{\mathrm{L}}(\mathcal{T})\right) \rightarrow\{\mathcal{V} \in \text { tors } \mathcal{A} \mid[\mathcal{V}, \mathcal{T}] \text { is a wide interval }\}
$$

We end this section applying our results to $\tau$-tilting theory. For this purpose, we recall some related notions.

Let $A$ be a finite-dimensional algebra over a field $K, M \in \bmod A$, and $P \in \operatorname{proj} A$. Then, the pair $(M, P)$ is called a support $\tau$-tilting pair if $(M, P)$ is $\tau$-rigid and $|M|+|P|=|A|$, where $|\cdot|$ denotes the number of isoclasses of indecomposable direct summands. Adachi-Iyama-Reiten [AIR, Theorem 2.7] showed that there exists a bijection from the set of basic support $\tau$-tilting pairs to the set of functorially finite torsion classes in $\bmod A$, given by $M \mapsto \mathrm{Fac} M$.

If two distinct support $\tau$-tilting pairs $(M, P) \neq\left(M^{\prime}, P^{\prime}\right)$ has a common direct summand $(N, Q)$ with $|N|+|Q|=|A|-1$, then we say that $\left(M^{\prime}, P^{\prime}\right)$ is a mutation of $(M, P)$. In this case, Fac $M^{\prime} \subsetneq$ Fac $M$ or Fac $M^{\prime} \supsetneq$ Fac $M$ holds AIR, DefinitionProposition 2.28]. The former case is called a left mutation, and the latter is called a right mutation.

For a fixed support $\tau$-tilting $(M, P)$, [DIJ, Theorem 3.1] implies that any arrow starting at Fac $M$ in Hasse(tors $\mathcal{A})$ comes from some left mutation of $(M, P)$; more explicitly, if $\mathcal{U} \in$ tors $\mathcal{A}$ has an arrow Fac $M \rightarrow \mathcal{U}$ in Hasse(tors $\mathcal{A}$ ), then there exists a left mutation of $\left(M^{\prime}, P^{\prime}\right)$ of $(M, P)$ satisfying Fac $M^{\prime}=\mathcal{U}$. Therefore the arrows starting at Fac $M$ in Hasse(tors $\mathcal{A}$ ) bijectively correspond to the left mutations of $(M, P)$. By Proposition 6.5, the labels of the arrows starting at Fac $M$ coincides with $\operatorname{sim}\left(\mathrm{W}_{\mathrm{L}}(\mathcal{T})\right)$. Thus we get the following result by using Theorem 6.7

Corollary 6.8. Let $A$ be a finite-dimensional algebra over a field $K$ and $(M, P)$ be a support $\tau$-tilting pair in $\bmod A$. Consider the torsion class $\mathcal{T}:=$ Fac $M$. If $m$ is the number of left mutations of the support $\tau$-tilting pair $(M, P)$, then $\operatorname{sim}\left(\mathrm{W}_{\mathrm{L}}(\mathcal{T})\right.$ ) has exactly $m$ elements, and there exist exactly $2^{m}$ torsion classes $\mathcal{V}$ such that $[\mathcal{V}, \mathcal{T}]$ are wide intervals.

We remark that all such torsion classes $\mathcal{V}$ are functorially finite in $\bmod A$ by JJas, Theorem 3.14].

\section{WIDELY GENERATED TORSION CLASSES}

In this section we consider widely generated torsion classes defined as follows:

Definition 7.1. Let $\mathcal{T} \in$ tors $\mathcal{A}$. Then $\mathcal{T}$ is called a widely generated torsion class if $\mathcal{T}$ admits some $\mathcal{W} \in$ wide $\mathcal{A}$ such that $\mathcal{T}=\mathrm{T}(\mathcal{W})$. 
By Proposition 6.1 it is easy to see that $\mathcal{T}$ is a widely generated torsion class if and only if $\mathcal{T}=\mathrm{T}\left(\mathrm{W}_{\mathrm{L}}(\mathcal{T})\right)$. We have more characterizations of widely generated torsion classes from the results in the previous section.

Theorem 7.2. For $\mathcal{T} \in$ tors $\mathcal{A}$, the following conditions are equivalent:

(a) $\mathcal{T}$ is a widely generated torsion class.

(b) $\mathcal{T}=\mathrm{T}\left(\mathrm{W}_{\mathrm{L}}(\mathcal{T})\right)$.

(c) $\mathcal{T}=\mathrm{T}(\mathcal{L})$ where $\mathcal{L}:=$ Label $[0, \mathcal{T}]^{+}$.

(d) For every $\mathcal{U} \in$ tors $\mathcal{A}$ such that $\mathcal{U} \subsetneq \mathcal{T}$, there exists a Hasse arrow $\mathcal{T} \rightarrow \mathcal{U}^{\prime}$ such that $\mathcal{U} \subseteq \mathcal{U}^{\prime}$.

Proof. (a) $\Leftrightarrow$ (b) follows from Proposition 6.1 and (b) $\Leftrightarrow$ (c) is implied by Proposition 6.5 .

(c) $\Rightarrow(\mathrm{d})$ : Let $\mathcal{U} \in$ tors $\mathcal{A}$ such that $\mathcal{U} \subsetneq \mathcal{T}$. Then there exists some $S \in \mathcal{L}$ such that $S \notin \mathcal{U}$ since $\mathcal{T}=\mathrm{T}(\mathcal{L})$. From there, one can conclude $\mathcal{U} \subseteq \mathcal{U}^{\prime}:=\mathcal{T} \cap{ }^{\perp} S$; indeed for every $U \in \mathcal{U}$ and $f: U \rightarrow S, f$ must be zero or epic by Lemma 6.4, and $f=0$ since $S \notin \mathcal{U}$.

(d) $\Rightarrow$ (c): The inclusion $\mathrm{T}(\mathcal{L}) \subseteq \mathcal{T}$ follows from $\mathcal{L} \subseteq \mathcal{T}$. For the other inclusion, suppose $\mathrm{T}(\mathcal{L}) \subsetneq \mathcal{T}$. Then by assumption there is a Hasse arrow $\mathcal{T} \stackrel{S}{\rightarrow} \mathcal{U}^{\prime}$ with $\mathrm{T}(\mathcal{L}) \subseteq \mathcal{U}^{\prime}$. Obviously we get $S \in \mathcal{L} \subseteq \mathrm{T}(\mathcal{L})$, but it contradicts $S \in\left(\mathcal{U}^{\prime}\right)^{\perp} \subseteq$ $\mathrm{T}(\mathcal{L})^{\perp}$.

Remark 7.3. The equivalences of (a), (c) and (d) above were also obtained in BCZ, Subsection 3.2] in terms of minimal extending modules and canonical join representations of torsion classes. If the conditions above hold, then $\mathcal{T}=\bigvee_{S \in \mathcal{L}} \mathrm{T}(S)$ is the canonical join representation of $\mathcal{T}$.

Example 7.4. Consider the following algebra $A$ appearing in Asa1, Example 4.13]:

$$
K(1 \underset{\beta}{\stackrel{\alpha}{\longrightarrow}} 2 \stackrel{\gamma}{\longrightarrow} 3) /\langle\alpha \gamma\rangle .
$$

Let $\mathcal{T}$ be the full subcategory

$$
\operatorname{add}\left(S_{3}, S_{1},{ }_{2}{ }_{2}{ }_{1}{ }_{2}{ }_{2}{ }_{2}, \ldots\right),
$$

where $S_{3}$ is the simple projective module corresponding to the vertex 3 , and $S_{1},{ }_{2}{ }_{2}{ }_{1}{ }_{2}{ }_{2}{ }^{1}, \ldots$ are all the indecomposable preinjective modules over the quotient algebra $A /\left\langle e_{3}\right\rangle \cong K(1 \rightrightarrows 2)$. Then one can check that $\mathcal{T}$ is a torsion class in $\bmod A$.

The simple projective module $S_{3}$ belongs to $\mathrm{W}_{\mathrm{L}}(\mathcal{T})$, and clearly it is simple in $\mathrm{W}_{\mathrm{L}}(\mathcal{T})$. There exists no other simple object in $\mathrm{W}_{\mathrm{L}}(\mathcal{T})$; indeed if $S \neq S_{3}$ is a simple object in $\mathrm{W}_{\mathrm{L}}(\mathcal{T})$, then $S$ must be a preinjective $\left(A /\left\langle e_{3}\right\rangle\right)$-module in $\mathcal{T}$, but one can easily check that any preinjective $\left(A /\left\langle e_{3}\right\rangle\right)$-module cannot be in $\mathrm{W}_{\mathrm{L}}(\mathcal{T})$. Thus $\mathrm{W}_{\mathrm{L}}(\mathcal{T})=$ Filt $S_{3}$, and $\mathcal{T} \neq \mathrm{T}\left(\mathrm{W}_{\mathrm{L}}(\mathcal{T})\right)$ follows. The condition (b) in Theorem 7.2 does not hold for the torsion class $\mathcal{T}$. Therefore $\mathcal{T}$ is not a widely generated torsion class.

Set $\mathcal{U}:=\mathcal{T} \cap{ }^{\perp} S_{3}$. Then the modules in $\mathcal{U}$ are all the preinjective $\left(A /\left\langle e_{3}\right\rangle\right)$ modules. There exists a Hasse arrow $\mathcal{T} \rightarrow \mathcal{U}$, and it is the unique Hasse arrow starting at $\mathcal{T}$ by Proposition 6.5. Thus $\mathcal{T}$ does not satisfy the condition (d) in Theorem 7.2 , for example add $S_{3} \subsetneq \mathcal{T}$ is a torsion class not contained in $\mathcal{U}$.

\section{FUNDING}

Sota Asai was supported by Japan Society for the Promotion of Science KAKENHI JP16J02249 and JP19K14500. 


\section{ACKNOWLEDGEMENT}

The authors thank Aaron Chan, Laurent Demonet, Osamu Iyama, Gustavo Jasso and Jan Schröer for kind instructions and discussions.

\section{REFERENCES}

[AIR] T. Adachi, O. Iyama, I. Reiten, $\tau$-tilting theory. Compos. Math. 150 (2014), no. 3, $415-452$.

[Asa1] S. Asai, Semibricks, to appear in Int. Math. Res. Not., https://academic.oup.com/imrn/advance-article/doi/10.1093/imrn/rny150/5049384

[Asa2] S. Asai, The wall-chamber structures of the real Grothendieck groups, in preparation.

[BCZ] E. Barnard, A. Carroll, S. Zhu, Minimal inclusions of torsion classes, arXiv: $1710.08837 \mathrm{v} 1$.

[BKT] P. Baumann, J. Kamnitzer, P. Tingley, Affine Mirković-Vilonen polytopes, Publ. Math. Inst. Hautes Études Sci. 120 (2014), 113-205.

[BB] S. Brenner, M. C. R. Butler, Generalizations of the Bernšteĭn-Gel'fand-Ponomarev reflection functors, Representation theory, II (Proc. Second Internat. Conf., Carleton Univ., Ottawa, Ont., 1979), 103-169, Lecture Notes in Math. 832, Springer, Berlin-New York, 1980.

[Bri1] T. Bridgeland, Stability conditions on triangulated categories, Ann. of Math. (2) 166 (2007), no. 2, 317-345.

[Bri2] T. Bridgeland, Scattering diagrams, Hall algebras and stability conditions, Algebr. Geom. 4 (2017), no. 5, 523-561.

[Brü] K. Brüning, Thick subcategories of the derived category of a hereditary algebra, Homology Homotopy Appl. 9 (2007), no. 2, 165-176.

[BST] T. Brüstle, D. Smith, H. Treffinger, Wall and Chamber Structure for finite-dimensional Algebras, arXiv:1805.01880v1.

[BY] T. Brüstle, D. Yang, Ordered exchange graphs, Advances in representation theory of algebras, 135-193, EMS Ser. Congr. Rep., Eur. Math. Soc., Zürich, 2013.

[DIJ] L. Demonet, O. Iyama, G. Jasso, $\tau$-tilting finite algebras, bricks, and g-vectors, Int. Math. Res. Not. IMRN 2019, Issue 3, 852-892.

[DIRRT] L. Demonet, O. Iyama, N. Reading, I. Reiten, H. Thomas, Lattice theory of torsion classes, arXiv:1711.01785 2 .

[Dic] S. E. Dickson, A torsion theory for Abelian categories, Trans. Amer. Math. Soc. 121 (1966), No. 1, 223-235.

[Hap] D. Happel, Triangulated Categories in the Representation Theory of Finite Dimensional Algebras, London Mathematical Society Lecture Note Series, 119, Cambridge University Press, 1988.

[HRS] D. Happel, I. Reiten, S. O. Smalø, Tilting in abelian categories and quasitilted algebras, Mem. Amer. Math. Soc. 120 (1996), no. 575.

[Hov] M. Hovey, Classifying subcategories of modules, Trans. Amer. Math. Soc. 353 (2001), no. $8,3181-3191$.

[IT] C. Ingalls, H. Thomas, Noncrossing partitions and representations of quivers, Compos. Math. 145 (2009), no. 6, 1533-1562.

[IRRT] O. Iyama, N. Reading, I. Reiten, H. Thomas, Lattice structure of Weyl groups via representation theory of preprojective algebras, Compos. Math. 154 (2018), no. 6, 12691305.

[Jas] G. Jasso, Reduction of $\tau$-tilting modules and torsion pairs. Int. Math. Res. Not. IMRN 2015, no. 16, 7190-7237.

[Kin] A. D. King, Moduli of representations of finite dimensional algebras, Quart. J. Math. Oxford Ser. (2) 45 (1994), no. 180, 515-530.

$[\mathrm{KY}] \quad$ S. Koenig, D. Yang, Silting objects, simple-minded collections, t-structures and co-tstructures for finite-dimensional algebras, Doc. Math. 19 (2014), 403-438.

[MŠ] F. Marks, J. Šťovíček, Torsion classes, wide subcategories and localisations, Bull. London Math. Soc. 49 (2017), Issue 3, 405-416.

[Miz] Y. Mizuno, Classifying $\tau$-tilting modules over preprojective algebras of Dynkin type, Math. Z. 277 (2014), no. 3-4, 665-690.

[Ric] J. Rickard, Morita theory for derived categories, J. Lond. Math. Soc. (2) 39 (1989), Issue 3, 436-456.

[Rin] C. M. Ringel, Representations of K-species and bimodules, J. Algebra 41 (1976), no. 2, 269-302.

[Yur] T. Yurikusa, Wide Subcategories are Semistable, Doc. Math. 23 (2018), 35-47. 
Sota Asai: Research Institute for Mathematical Sciences, Kyoto University, KitashirakawaOiwakecho, Sakyo-Ku, Kyoto-Shi, Kyoto-Fu, 606-8502, Japan

E-mail address: asaisota@kurims.kyoto-u.ac.jp

Calvin Pfeifer: Mathematisches Institut, Universität Bonn, Endenicher Allee 60, 53115 Bonn, Germany

E-mail address: calvin.pfeifer@uni-bonn.de 